\title{
Characterizing well-differentiated culture of primary human nasal epithelial cells for use in wound healing assays
}

\author{
Johanna Schagen ${ }^{1} \cdot$ Peter D. Sly $^{1} \cdot$ Emmanuelle Fantino $^{1}$
}

Received: 24 January 2018 / Revised: 31 May 2018 / Accepted: 5 June 2018 / Published online: 8 August 2018

(c) United States \& Canadian Academy of Pathology 2018

\begin{abstract}
The nasal epithelium is the initial contact between the external environment and the respiratory tract and how it responds to noxious stimuli and repairs epithelial damage is important. Growing airway epithelial cells in culture at air-liquid interface allows for a physiologically relevant model of the human upper airways. The aim of the present study was to characterize human primary nasal epithelial cells grown at the air-liquid interface and establish a model for use in wound healing assays. This study determined the time required for full differentiation of nasal epithelial cells in an air-liquid interface culture to be at least 7 weeks using the standardized B-ALI media. Also, a model was established that studied the response to wounding and the effect of EGFR inhibition on this process. Nasal epithelial cultures from healthy subjects were differentiated at airliquid interface and manually wounded. Wounds were monitored over time to complete closure using a time lapse imaging microscope with cultures identified to have a rate of wound healing above $2.5 \% / \mathrm{h}$ independent of initial wound size. EGFR inhibition caused the rate of wound healing to drop a significant $4.6 \% / \mathrm{h}$ with there being no closure of the wound after $48 \mathrm{~h}$. The robust model established in this study will be essential for studying factors influencing wound healing, including host disease status and environmental exposures in the future.
\end{abstract}

\section{Introduction}

The airway epithelium provides the first line of innate immune protection that includes anti-oxidant defence and orchestrating an inflammatory response against respiratory viruses and bacteria [1]. It also plays important roles in modulation of adaptive immunity, mucociliary clearance, with production of antimicrobial peptides and proteins, reactive oxygen species and release of cytokines, chemokines, and growth factors [2, 3]. A critical role for the epithelium in maintaining homeostasis is the ability to provide a physical barrier protecting against invading microorganisms and noxious stimuli. An important part of this barrier function is the formation of tight junctions and the ability to repair epithelial injury rapidly.

Peter D. Sly

p.sly@uq.edu.au

1 Children's Lung, Environment and Asthma Research Team, Centre for Children's Health Research, The University of Queensland, Brisbane, Australia
Previous studies using a monolayer of cultured epithelial cells harvested from both nasal and bronchial sites have demonstrated important aspects of wound healing from migration and proliferation of basal epithelial cells $[4,5]$. However, submerged monolayer cultures lack many features of the normal epithelium such as mucociliary differentiation [6]. Growing epithelial cells at the air-liquid interface (ALI) allows for differentiation into a pseudostratified epithelium with basal cells, cilia projecting from the apical surface, and goblet cells producing mucus [7, 8]. Many studies confirm ALI as a physiologically relevant platform for studying epithelial function and mechanisms associated with respiratory diseases and represents an excellent and more realistic model for airway epithelial function experiments [9-15]. Differentiation of nasal epithelial cells at ALI takes at least 21 days after "lift" as recommended by the media manufactures [16] but much conjecture about this differentiation period exists in the literature. Studies have used ALI cultures from as early as 14 days for human rhinovirus infections [17] with cells harvested from the trachea and nose, to as late as 42 days to see mucus production [17] and cilia beat frequency from nasal cells [18]. These studies harvested bronchial epithelial cells from the trachea whereas this study proposes to use 
epithelial cells harvested from the nasal cavity. Ong et al. [19] has already reported that nasal epithelial cells take longer to differentiate ( 7 weeks) than bronchial epithelial cells (3 weeks) and explains the wide differences seen in time it takes to form a pseudostratified epithelial culture at ALI.

Mechanisms underlying epithelial growth and repair have been studied in submerged monolayer culture [20, 21] but not yet in ALI cultures. Epidermal growth factor (EFG), signalling through the epidermal growth factor receptor (EGFR), regulates cell division and death but also normal differentiation, mucus production and mediates repair in epithelial cells [22, 23]. Normal function through the receptor allows for activation of the MAPK and PI3K pathways that are essential in cell proliferation and cell survival. Erlotinib, an inhibitor of EGFR, binds to the tyrosine kinase domain instead of ATP causing a reduction in cell proliferation and cell survival [24]. Data are lacking of the effect of EGFR on epithelial cells grown in ALI culture.

The nasal epithelium represents the point of first contact between the external environment and the respiratory tract. As such, how the nasal epithelium respond to noxious stimuli and repairs epithelial damage is important. The aim of the present study was to characterize human primary nasal epithelial cells, grown at the ALI, for use in wound healing assays. We determined the time required for full differentiation of the epithelium, developed methods for studying the response to wounding and the effect of EGFR inhibition on this process.

\section{Materials and methods}

Primary human nasal epithelial cells were collected from 18 adult non-atopic healthy adult subjects aged 18-55 years old. From these 18, only 13 were successful in ALI culture with three used for determination of full differentiation experiments and ten for the wound healing assay. Cells were isolated from the epithelial layer lining the nasal cavity by scraping the anterior surface of the inferior turbinate using a purpose designed curette (ASI Rhino-Pro, Arlington Scientific, USA) [25]. The project was approved by the human research ethics committees of Children's Health Queensland (2011000058) and of The University of Queensland (2017000520). All volunteers gave written consent for the study.

\section{Cell culture}

Cells were grown in submerged culture for 3 weeks until they reached passage 2 , harvested and cryopreserved for later use. When required, cells were thawed and seeded $(25,000$ cells/insert $)$ on collagen coated Transwell permeable polyester membrane inserts with a $0.4 \mu \mathrm{m}$ pore size in 24 well plates (Corning Costar, Cambridge, MA, USA) and grown in Bronchial Epithelial Cell Growth Medium (BEGM, Lonza). After approximately three days of cell division the cells are "lifted" with media removed from the apical chamber and media in the basal chamber replaced with B-ALI ${ }^{\mathrm{TM}}$ Bronchial Air Liquid Interface BulletKit $^{\mathrm{TM}}$ (B-ALI, Lonza) [26]. Cells were maintained for at least 3 weeks until a pseudostratified epithelium with a high transepithelial electrical resistance (TEER) was established. A successful ALI culture contains basal cells, tight junctions, goblet cells, and ciliated epithelial cells in which the cilia are seen to be beating. Cilia were observed under light microscope and detected by their movement.

\section{Transepithelial electrical resistance}

The TEER value was measured using the EVOM2 machine (World Precision Instruments). TEER was measured before the start of each experiment and at each time point before sample collection and change of media. TEER was calculated by subtracting the resistance of a blank insert then using the formula as follows to correct for the surface area of the insert:

$$
\Omega . \mathrm{cm}^{2}=\Omega(\text { OHMS }) \times \text { Transwell area insert }\left(0.33 \mathrm{~cm}^{2}\right)
$$

\section{Time to full differentiation}

ALI cultures were maintained using B-ALI media, with hydrocortisone, for a period of 7 weeks with TEER values recorded at 3, 5, and 7 weeks. Wells were harvested at each time point for immunofluorescence staining and confocal imaging.

\section{Immunofluorescence staining and confocal imaging}

Epithelial ALI cell cultures were washed with PBS three times and fixed in paraformaldehyde (3\%) for $10 \mathrm{~min}$ at room temperature and rinsed in PBS. Cell membranes were permeabilized with Triton X100 (0.5\% in PBS) for $10 \mathrm{~min}$ at room temperature and blocked with BSA (2\%) in Triton X100 (0.2\%) for $1 \mathrm{~h}$ at room temperature. Cells were incubated with primary antibodies at $4{ }^{\circ} \mathrm{C}$ overnight and washed three times with Triton X100 (0.5\% in PBS). Secondary antibodies were added and incubated for $1 \mathrm{~h}$ at $37^{\circ} \mathrm{C}$. After two washes with Triton X100 $(0.5 \%$ in PBS) and PBS each, cells were stained with DAPI (Hoechst) for 10 min before mounting with Prolong Gold Antifade reagent (Life Tech). Anti-ZO-1/TJP1 (40-2200) antibody and Acetyl-alpha-tubulin (32-2700) antibody were purchased from Thermo Scientific. Anti-MUC5B 
Table 1 Summary of the observed fluorescence staining of the epithelial cells at ALI over a 7 week differentiating period

\begin{tabular}{llllllll}
\hline $\begin{array}{l}\text { Subject } \\
\text { lift }\end{array}$ & $\begin{array}{l}\text { Days to } \\
\text { lift }\end{array}$ & $\begin{array}{l}\text { Weeks post } \\
\left(\Omega \mathrm{cm}^{2}\right)\end{array}$ & $\begin{array}{l}\text { TEER } \\
\text { lifiliated cells }\end{array}$ & $\begin{array}{l}\text { Basal } \\
\text { cells }\end{array}$ & $\begin{array}{l}\text { Goblet } \\
\text { cells }\end{array}$ & $\begin{array}{l}\text { Tight } \\
\text { junctions }\end{array}$ \\
\hline \multirow{4}{*}{14} & 1 & 3 & 65 & $\boldsymbol{x}$ & $\checkmark$ & $\checkmark$ & $\checkmark$ \\
& & 5 & 610 & $\boldsymbol{x}$ & $\checkmark$ & $\checkmark$ & $\checkmark$ \\
& & 7 & 461 & $\checkmark$ & $\checkmark$ & $\checkmark$ & $\checkmark$ \\
15 & 3 & 252 & $\boldsymbol{x}$ & $\checkmark$ & $\checkmark$ & $\checkmark$ \\
& & 5 & 772 & $\boldsymbol{x}$ & $\checkmark$ & $\checkmark$ & $\checkmark$ \\
& & 7 & 291 & $\checkmark$ & $\checkmark$ & $\checkmark$ & $\checkmark$ \\
& & 3 & 43 & $\boldsymbol{x}$ & $\checkmark$ & $\checkmark$ & $\checkmark$ \\
\hline
\end{tabular}

Presence or absence of immunofluorescence stain for three subjects. Ciliated cells were only seen for all subjects at week 7
(HPA008246) was purchased from Sigma and anticytokeratin 14 (ab192694) purchased from Abcam. Alexa fluor 594 goat anti-mouse, alexa fluor 488 chicken antirabbit, alexa fluor 594 goat anti-rabbit and alexa fluor 488 goat anti-guinea pig obtained from Life Technology (Carlsbad, CA). Images were captured from two fields per well at 20x magnification with a Nikon inverted fluorescent microscope (Model HB-10101AF), filters suitable for DAPI (blue), 488 (green), and 594 (red) fluorescence were used with a Spot CCD camera (Model 1.1.0).

\section{Wound healing assay}

Fully differentiated ALI cultures were starved of hydrocortisone in basolateral media for three days prior to wounding. Mechanical injury was performed by scraping a P10 sterile pipette tip across the cell layer creating a wound with a diameter ranging from 600 to $1500 \mu \mathrm{m}$. After wounding, the apical surface of the culture was washed with $200 \mu \mathrm{L}$ PBS to remove cellular debris. The leading edge of the wound was tracked with the aid of time-lapse microscopy (CytoSmart, Lonza) with images taken every $60 \mathrm{~min}$ at $4 \times$ magnification and image analysis software used to track the wound area over time. Image editing software (Adobe Photoshop) was used to remove the wound area and convert image files to .TIFF. Wound area was calculated each hour using ImageJ by converting pixels to area, where one pixel $=2 \mu \mathrm{m}^{2}$. The initial wound area was expressed as $100 \%$ to remove variability from wounds of different size. Wounds were considered to be closed when the calculated area fell below 3\%, the effective limit of detection of the image processing. Wound closure was calculated as follows:

Wound closure $(\%)=100-(($ Area/Initial area $) * 100)$

Wound closure (\%), plotted as a function of time (h), was used to calculate the rate of wound closure $(\% / \mathrm{h})$.

\section{Cytokine}

Supernatants of epithelial ALI cells were collected by washing the apical surface of the ALI culture with PBS at pre-wounding and $48 \mathrm{~h}$. Washes were tested for cytokine detection using AlphaLISA kit (Perkin Elmer) following manufacturer's recommendations. Cytokines tested: GMCSF (AL216C, dynamic range 1.6-10,000 pg/mL), IL-13 (AL24OC, dynamic range 3.0-100,000 pg/mL), and IP-10/ CXCL10 (AL326C, dynamic range 3.3-300,000 pg/mL).

\section{EGFR inhibition}

Erlotinib $\mathrm{HCl}(2 \mu \mathrm{g} / \mathrm{mL})(\mathrm{OSI}-744$, Shelleckchem) [27] was added to the basolateral media 7 days prior to wounding and replenished every two days when media was changed.

\section{Statistics}

Data were analyzed with GraphPad Prism 7.0 (GraphPad Inc., La Jolla, CA, USA) using paired $t$-test and correlation, as appropriate, with a $p<0.05$ taken to be statistically significant.

\section{Results}

\section{Time to full differentiation}

Epithelial cells were grown at ALI for 7 weeks and stained for the presence of tight junctions with basal, goblet and ciliated cells. Three ALI cultures were stained at 3, 5, and 7 weeks post air-lift. Tight junctions, basal cells and goblet cells were present at 3 weeks in all cultures (Table 1). Ciliated cells appeared by week 7 in two subjects but were not seen in the third (Table 1). Representative data are shown in Figs. 1, 2. The TEER value for all three subjects shows an increase from week 3 to week 7 with a peak at week 5 (Table 1). 


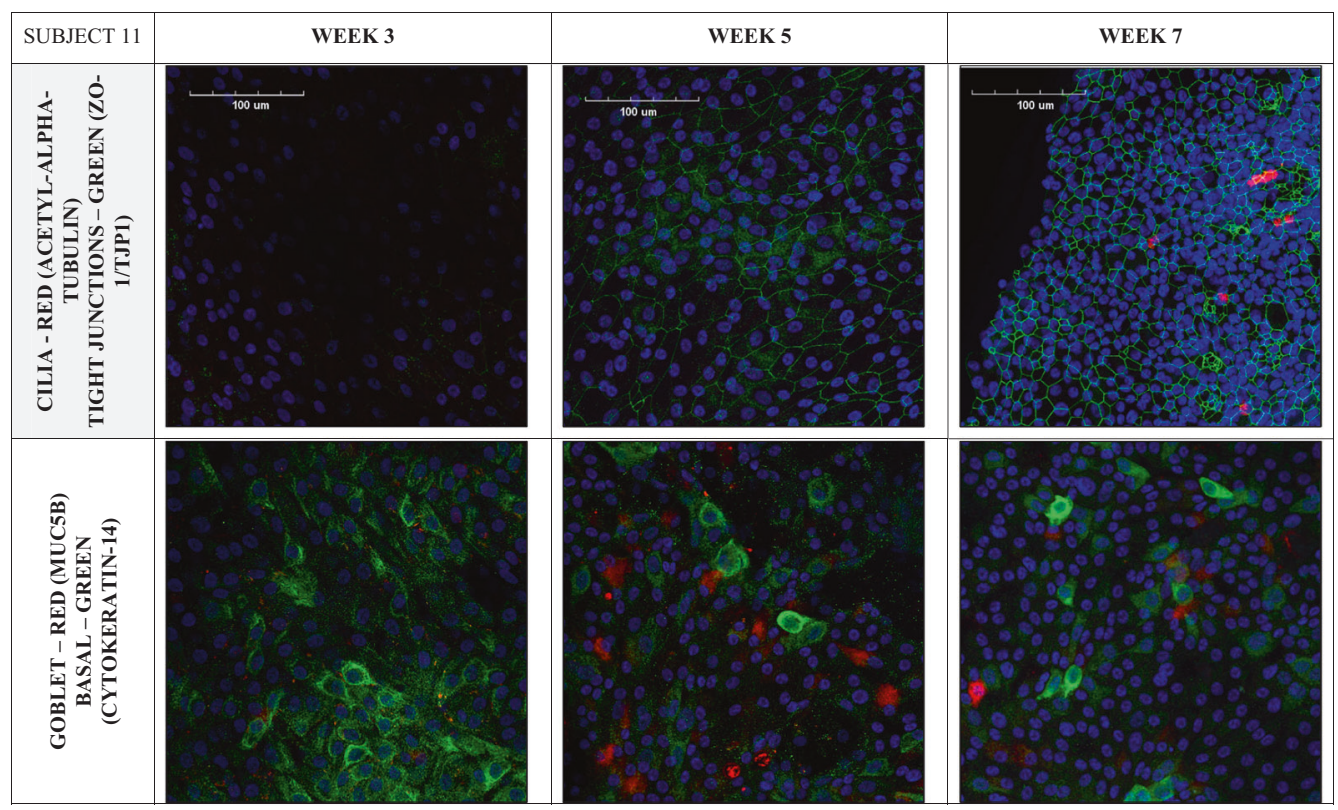

Fig. 1 Cells grown at ALI in B-ALI media for 7 weeks. Cultures subjected to immunofluorescence labelling as in 'Methods'. Cultures were labelled with basal (cytokeratin 14), goblet (MUC5B) and

\section{In vitro model for airway epithelium wound healing}

The average rate of wound closure for three subjects showed $4.34 \% \pm 0.75 / \mathrm{h}$ for Subject 1 and $3.09 \% \pm 0.37 / \mathrm{h}$ for Subject 2 both after 1 repeat, and $3.89 \% \pm 1.08 / \mathrm{h}$ for Subject 8 after two repeats (data not shown).

\section{Rate of wound closure}

Wound closure was complete within $35 \mathrm{~h}$ for $9 / 10(91.7 \%)$ of cultures (Fig. 3, Table 2). The group mean rate of healing for those subjects was $4.3 \% / \mathrm{h}(\mathrm{SD}=1.3 \% / \mathrm{h})$, with all "healing" subjects achieving a rate above $2.5 \% / \mathrm{h}$ (Table 2 ). One non-healing subject had a rate of $0.73 \% / \mathrm{h}$ (Table 2). There was no correlation between the rate of epithelial rate of wound closure in healthy and initial wound size $\left(\mu \mathrm{m}^{2}\right)$ with $r^{2}=0.06$ (Fig. 4).

\section{Cytokines released}

Detection levels of IL-13, GM-CSF, and IP-10 showed no difference before wounding and after wound closure with levels at the lower detection limits for the assays (Fig. 5).

\section{Erlotinib exposure}

Exposure to erlotinib 7 days prior to wounding caused a significant decrease in the rate of wound closure from $5.06 \% / \mathrm{h} \quad(\mathrm{SD}=1.48 \% / \mathrm{h}) \quad$ to $0.43 \% / \mathrm{h} \quad(\mathrm{SD}=0.07 \% / \mathrm{h})$ (Fig. 6). ciliated (Acetyl-alpha-tubulin) cells and tight junctions (ZO-1/TJP1) with DAPI staining. Representative images form Subject 11 are shown. Scale bar represents $100 \mu \mathrm{m}$

\section{Discussion}

The differentiation of a pseudostratified epithelium at ALI allows for greater understanding of the cell-to-cell interactions and provides a unique opportunity to study epithelial cells in vitro. In the present study, we analysed healthy adult epithelial cells at ALI to determine the ideal timing and conditions needed for a representative model of wound healing and repair. The results demonstrated rapid wound healing within $36 \mathrm{~h}$ that could be characterized by a linear rate using image analysis software.

\section{Full differentiation takes longer than often reported}

The culture conditions used to differentiate epithelial cells into pseudostratified epithelial layer, containing goblet, basal, and ciliated cells vary in the literature. We chose to use the standardized B-ALI media from Lonza because of its concentration controlled additives and its commercial availability. For BALI media, hydrocortisone is an additive of the media that is included and suggested by manufactures. We have excluded it just from the wound healing experiments because hydrocortisone treatment is effective at reducing inflammatory immune reaction and airway hyperresonsiveness [28]. Hydrocortisone was present for the full differentiation period but removed from the basal media three days prior wounding. There was no difference seen in the health of the culture when hydrocortisone was removed (data not included). Numerous past studies have also used this media for differentiation of the airway 
Fig. 2 Wound closure assay in epithelial cells at ALI.

Representative photographs at $0 \mathrm{~h}, 16 \mathrm{~h}$, and $24 \mathrm{~h}$ after wounding in Subject 9 (a) with wound area identified (b) and removed (c) for ImageJ analysis

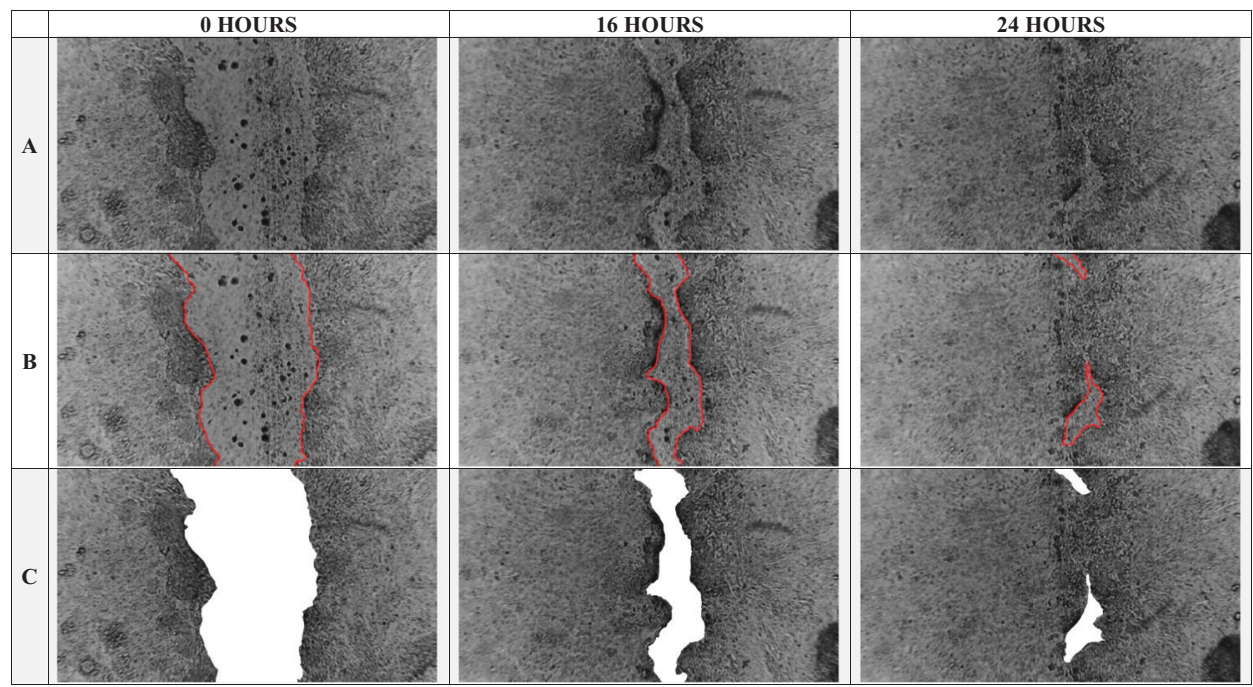

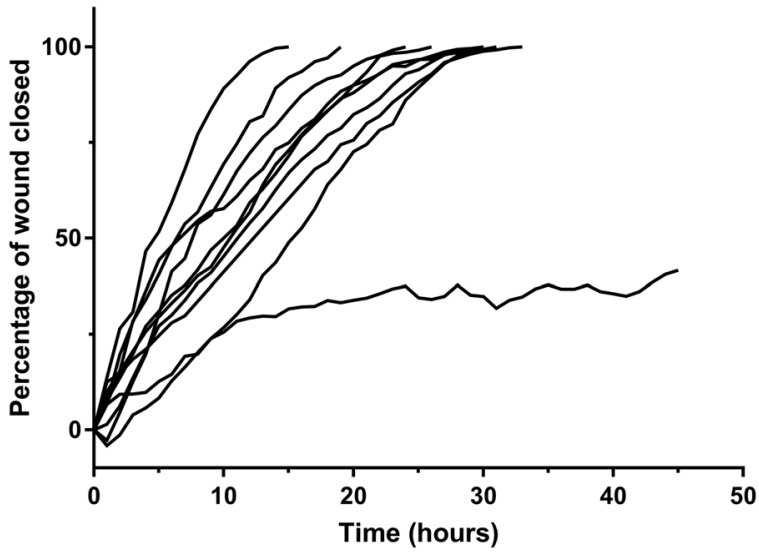

Fig. 3 Rate of wound closure. Leading edge of the wound was tracked and the area of the wound determined every $60 \mathrm{~min}$ until closure of wound. Wound areas were graphed for each participant as a percentage of wound closure (wound closed $=100 \%$ ) over time

epithelium but with experimentation at varying days post lift $[7,17,18,29]$. The reported days post lift vary from 14 to 42 days with the shortest reported by Hao et al., which is 7 days earlier than even the manufacture recommends $[17,18]$. The site epithelial cells are harvested from also plays a part in the time to full differentiation, with nasal epithelial cells reported to take much longer [19]. These differences can also be seen in a study by Comer et al. [30] where they have conducted experiments side-by-side using both nasal and bronchial epithelial cells. For our study of the epithelium we must first look at nasal epithelial cells as they are easier to obtain and can be used as an alternative to bronchial epithelial cells. These discrepancies identified a gap where standardized methods of growing nasal epithelial cells at the ALI needed to be established. In our lab with B-ALI media, 3 weeks after lift was insufficient, with a longer period needed for the ALI culture to differentiate into all three cell types. By staining ALI cultures over a 7 week period for basal, goblet, and ciliated cells with tight junctions we are able to confirm that only at 7 weeks post lift are we able to see all cell types, with ciliated cells only visible at that time in conjunction with a high TEER reading (Table 1). The reduction in the TEER from 5 to 7 weeks does not indicate a reduction in tight junctions. The development of cilia may be the cause of the lower TEER with more cell types present at that time. Ong et al. [19] reported that they also saw a decrease in TEER after 3 weeks in conjunction with the observation of cilia formation, with TEER only representing the resistance of the tight junctions. The lineage of each cell type and structure of the epithelium developed at differing rates over the 7 weeks. Basal cells develop early with studies identifying them as the progenitor cells of the epithelium [31]. They were seen from the earliest time point of 3 weeks post lift with studies confirming that the monolayer of submerged cells at seeding stage of ALI are basal cells [32]. Tight junctions were also present from week 3 but with development increasing by week 5 as confirmed by the high TEER value for all subjects (Table 1). Goblet cells were identifiable at 3 weeks post lift but became much more evident after 5 and 7 weeks (Table 1). Mechanisms involved in the differentiation of goblet cells are more complex and include the activation of the EGFR [23] and transcription factors, FOXA2 and SPDEF [33], resulting a longer differentiation period. The last cell type to differentiate in the ALI culture were ciliated cells. For two of the three subjects they were evident at 7 weeks post lift (Table 1) with more time presumably needed for cilis to occur in the remaining subject. In the airway epithelium, ciliated cells are essential for the transport of mucus and removal of external pathogens [34]. Waiting 7 weeks for cilia cells to develop in the ALI model is essential because of their importance in maintaining the upper airway homeostasis. 
Table 2 Characteristics of subjects

\begin{tabular}{|c|c|c|c|c|c|c|c|c|c|}
\hline \multirow[t]{2}{*}{ Subject } & \multirow[t]{2}{*}{ Sex } & \multicolumn{2}{|c|}{ TEER $\left(\Omega . \mathrm{cm}^{2}\right)$} & \multirow{2}{*}{$\begin{array}{l}\text { Time to } \\
\text { closure (h) }\end{array}$} & \multicolumn{2}{|c|}{ Wound area $\left(\mu \mathrm{m}^{2}\right), \%^{\mathrm{a}}$} & & \multirow{2}{*}{$\begin{array}{l}\text { Rate of } \\
\text { wound } \\
\text { closure } \\
(\% / \mathrm{h})\end{array}$} & \multirow[t]{2}{*}{$R$ squared } \\
\hline & & $\begin{array}{l}\text { Pre } \\
\text { wounding }\end{array}$ & $48 \mathrm{~h}$ & & Initial & Final & & & \\
\hline 1 & Female & 1402 & 643 & 25 & 586,748 & 4820 & $0.8 \%$ & 4.49 & 0.93 \\
\hline 2 & Male & 280 & 313 & 31 & 461,552 & 1790 & $0.4 \%$ & 3.36 & 0.95 \\
\hline 3 & Male & 577 & 478 & 16 & 457,662 & 1626 & $0.7 \%$ & 6.75 & 0.94 \\
\hline $4^{b}$ & Female & 1270 & 181 & 46 & 462,954 & 270058 & $58.3 \%$ & 0.73 & 0.67 \\
\hline 5 & Male & 1699 & 1699 & 27 & 335,258 & 2804 & $0.8 \%$ & 3.94 & 0.98 \\
\hline 6 & Female & 280 & 280 & 32 & 550,584 & 3532 & $0.6 \%$ & 3.58 & 0.98 \\
\hline 7 & Male & 280 & 280 & 21 & 307,284 & 8772 & $2.9 \%$ & 5.22 & 0.96 \\
\hline 8 & Female & 346 & 643 & 31 & 522,444 & 4378 & $0.8 \%$ & 3.47 & 0.97 \\
\hline 9 & Female & 1930 & 2293 & 32 & 432,494 & 3562 & $0.8 \%$ & 2.9 & 0.90 \\
\hline 10 & Female & 181 & 214 & 33 & 395,260 & 874 & $0.2 \%$ & 3.08 & 0.98 \\
\hline
\end{tabular}

No significant difference was seen between TEER values pre wounding and at $48 \mathrm{~h}$ ( $t$-test, $p$-value 0.42 ). Significant difference was seen between the initial and final wound area for all except subject 4 ( $t$-test, $p$-value $<0.0001)$

${ }^{a}$ Final wound area expressed as a percent with the initial wound area normalized to $100 \%$

${ }^{\mathrm{b}}$ Subject 4 was removed from further analysis as the wound failed to close

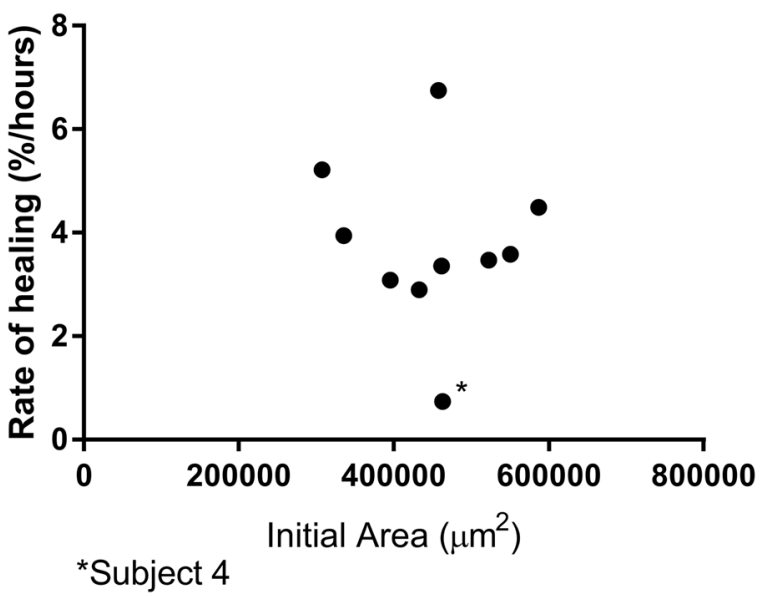

Fig. 4 Rate of healing $(\% / \mathrm{h})$ compared against the initial area $\left(\mu \mathrm{m}^{2}\right)$ of each wound. At time 0 , the area of the initial wound was calculated as initial area $\left(\mu \mathrm{m}^{2}\right)$. There was no correlation seen between initial area $\left(\mu \mathrm{m}^{2}\right)$ and rate of healing $(\% / \mathrm{h})$ for all donors at the $95 \%$ confidence interval

A pseudostratified columnar airway epithelium can be established in an ALI culture, however, for adult nasal primary cells this must be monitored on a subject-to-subject basis with a minimum 7 weeks required for full differentiation. This period allows for the development of basal, goblet, and ciliated cells with a stable TEER value. Authors should be encouraged to report the state of the ALI cultures used in their studies.

\section{Rate of closure is an appropriate outcome variable for studying influence on wound healing}

Previous reports on wound healing have used cells cultured in monolayer, submerged cultures with only basal cells present $[5,35]$. The present study used the method of scratch assays similar to these previous studies, adapted for ALI culture. The 3D nature of the ALI system creates obstacles in the wounding, imaging, and even sampling of the cultures. Common software programs previously used for analysis of monolayer scratch assays are not able to identify the wound edge (TScratch and Robust Quantitative Scratch Assay) in ALI cultures. The present study used simple programs such as Adobe Photoshop and ImageJ that allow identification and calculation of the wound area (Fig. 3). The initial wound size did not correlate with the rate of wound closure (Fig. 4). This allowed normalization of the initial wound to $100 \%$ with healing plotted as a percentage of the initial wound and the rate of wound closure taken from the slope (Fig. 3). Only one subject failed to close the wound and had a rate of wound healing less than $1 \% / \mathrm{h}$. From the remaining subjects that did close, we report a rate of wound healing of above $2.5 \% / \mathrm{h}$ (Table 2). Thus, we suggest $2.5 \% / \mathrm{h}$ as a baseline rate of wound healing for healthy subjects that can be used in future experiments investigating factors that disturb normal wound healing in the airway epithelium. The robustness of this wound healing assay was measured by repeated wound healing of the same subject on different wells and on different days, for three subjects. Each subject showed a minimal assay-to-assay variation $(<28 \%)$. All remained above the recommended $2.5 \% / \mathrm{h}$ allowing for confidence in the wound healing assay and ALI model.

Epithelial wound healing occurs by four processes: dedifferentiation, migration, proliferation, and redifferentiation [36-39]. The present study shows that ALI cultures of primary nasal epithelial cells from healthy subjects 

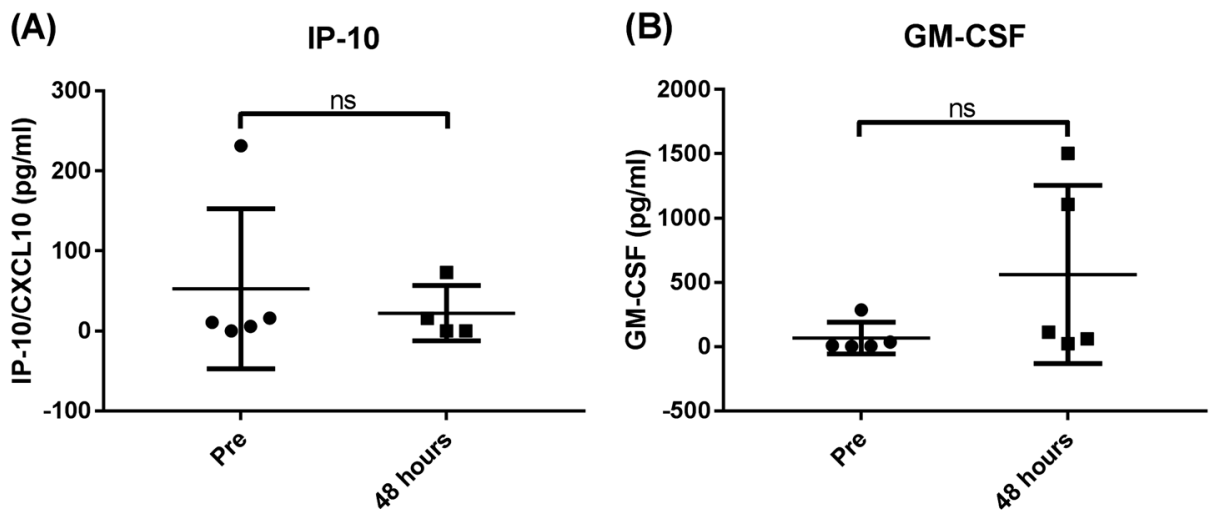

(C)

IL-13

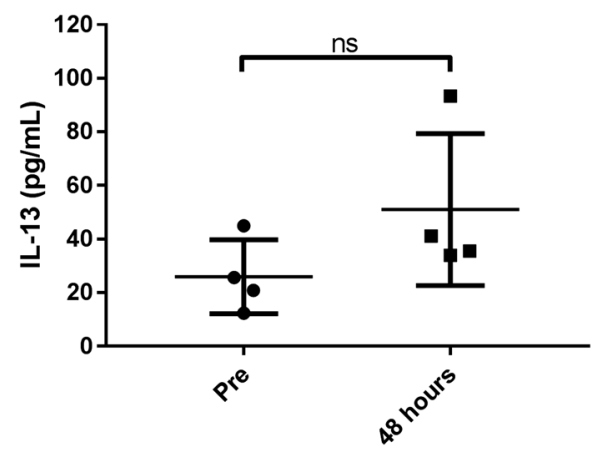

Fig. 5 Cytokine concentration before and after wound closure. Apical washes were taken at pre-wound, and $48 \mathrm{~h}$ when wound was closed. Samples were analysed IP-10 (a), GM-CSF (b), and IL-13 (c) (Subjects 1, 2, 3, 5, and 11). No significant difference was seen between before or after wounding for all three cytokines measured $(n=5)$. Note: no IL-13 was detected in subject 2

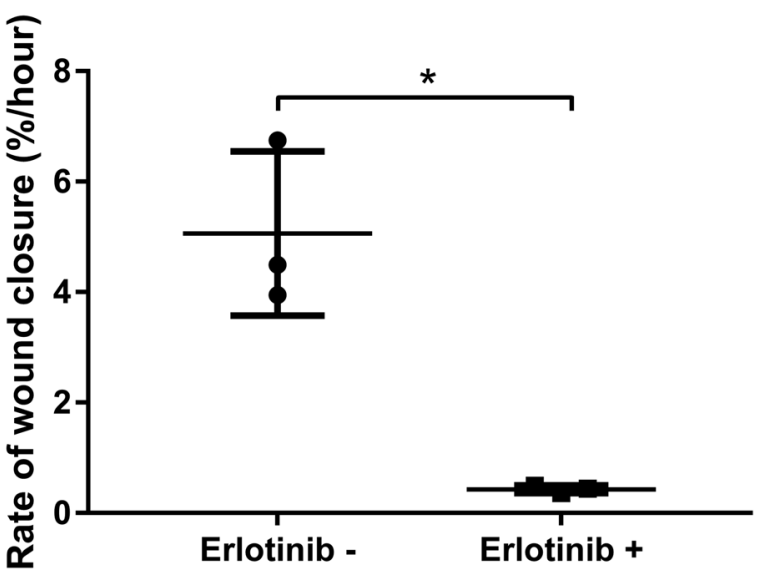

Fig. 6 Rate of wound closure for basolateral exposure. Erlotinib $(2 \mu \mathrm{g} / \mathrm{mL})$ was added to the basolateral media of ALI culture 7 days prior to wounding $(n=3)$. The rate of wound closure was significantly lower after erlotinib $(p=0.03)$

heal within $36 \mathrm{~h}$ (Fig. 3). However, this period may only represent the first two stages of wound repair, dedifferentiation and migration. Proliferation and redifferentiation are likely to occur once the wound is visually closed, but may be inferred from the TEER at $48 \mathrm{~h}$ post wounding using this model (Table 2). The use of fluorescence staining and cytokine release could confirm this. For eight subjects TEER returned to the around the pre-wounding value by $48 \mathrm{~h}$, which is likely to indicate that the epithelium has polarized with tight junction present and indicate complete repair of the wound.

Cytokine levels are a reliable and useful measurement of inflammation and innate immunity [40-42]. However, most previous reports used submerged monolayer cultures, where sampling of the culture is easier and less disruptive to the epithelial cells. The situation appears more complex with ALI cultures. In the present study, we chose to measure cytokines on the apical surface of the culture, as this is the most applicable site. However, one consequence of our decision was that we were unable to reliably sample during the healing process. There were no significant differences in the cytokine levels before wounding and after wound closure (Fig. 5) which is contrary to Allahverdian et al. [43], that show an increase in IL-13 during wound healing. There was also only a small number of donors tested with plans looking at increasing these numbers in the future. Although the cytokine levels from the apical wash were low, we were still able to detect epithelial release of GM-CSF, IP-10, and IL-13 (Fig. 5). GM-CSF is reported to be released by epithelial cells [42], although not widely 
reported. IP-10/CXCL10 is also of interest because it is produced by epithelial cells with an increased expression seen in respiratory diseases such as asthma and COPD after pathogen exposure [44]. This observation of no change seen before and after closure could be consistent with the model only showing dedifferentiation and migration only, and GM-CSF, IP-10 and IL-13 may not be involved in these processes. Other cytokines might be more suitable for quantification using this model including, IL-6 and IL-8. IL-6 is expressed in response to environmental stress factors including infections and tissue injuries [45], and IL- 8 has been shown to increase during cell adhesion and migration [46].

A functional EGF-EGFR pathway is critical for wound healing in the submerged monolayer culture system and of interest in our wound healing assay [47]. The results from the present study show that this is also critical for healing wounds in ALI culture (Fig. 6). EGFR is part of a pathway involved in the repair of damaged epithelium [47] and by blocking this pathway with erlotinib we saw a reduction in the proliferation and survival of epithelial cells [48], with a rate of healing $<1 \% / \mathrm{h}$ and failure to heal within $48 \mathrm{~h}$.

The present study used epithelial cells sampled from the nasal cavity of healthy adults. The nasal epithelium is the "site of first contact" for pathogens and environmental stimuli. Nasal airway epithelial cells are easily accessible and resemble the upper airway epithelium morphology with similar gene expression and mucus production [49]. We are deliberately studying the response of the nasal epithelium in its own right and not as a substitute for lower airway epithelium. As such, the results of the present study may not be applicable in the lower airways. However, as the structure and cell types are similar to those in the larger conducting airways, similar results are likely for cells obtained from those airways [50].

In conclusion, the results from the present study showed that well-differentiated cultures of primary nasal epithelial cells grown at the air-liquid interface are suitable for studying wound healing and that measuring the rate of wound healing gives a reliable outcome variable. The ALI culture must have the presence of basal, goblet, and ciliated cells with a stable TEER before wounding of the culture and show a decrease in the rate of wound healing after exposure to erlotinib. We plan to use this model to study factors influencing wound healing, including host disease status and environmental exposures.

Acknowledgements The authors of this paper would like to acknowledge the volunteers who participated in this study. This research was supported by The Australian Government Research Training Program Scholarship and The Asthma Foundation Queensland.

\section{Compliance with ethical standards}

Conflict of interest The authors declare that they have no conflict of interest.

\section{References}

1. Fulcher ML, Gabriel S, Burns KA, et al. Well-differentiated human airway epithelial cell cultures. Methods Mol Med. 2005; 107:183-206.

2. Bals R, Hiemstra PS. Innate immunity in the lung: how epithelial cells fight against respiratory pathogens. Eur Respir J. 2004;23: 327-33.

3. Parker D, Prince A. Innate immunity in the respiratory epithelium. Am J Respir Cell Mol Biol. 2011;45:189-201.

4. Wesley UV, Bove PF, Hristova M, et al. Airway epithelial cell migration and wound repair by ATP-mediated activation of dual oxidase 1. J Biol Chem. 2007;282:3213-20.

5. Perotin JM, Adam D, Vella-Boucaud J, et al. Delay of airway epithelial wound repair in COPD is associated with airflow obstruction severity. Respir Res. 2014;15:151.

6. Whitcutt MJ, Adler KB, Wu R. A biphasic chamber system for maintaining polarity of differentiation of cultured respiratory tract epithelial cells. Vitr Cell Dev Biol. 1988;24:420-8.

7. Ashraf S, Brockman-Schneider R, Bochkov YA, et al. Biological characteristics and propagation of human rhinovirus- $\mathrm{C}$ in differentiated sinus epithelial cells. Virology. 2013;436:143-9.

8. Ashraf S, Brockman-Schneider R, Gern JE. Propagation of rhinovirus-C strains in human airway epithelial cells differentiated at air-liquid interface. Methods Mol Biol. 2015;1221:63-70.

9. Krunkosky TM, Jordan JL, Chambers E, et al. Mycoplasma pneumoniae host-pathogen studies in an air-liquid culture of differentiated human airway epithelial cells. Microb Pathog. 2007; 42:98-103.

10. Thaikoottathil JV, Martin RJ, Zdunek J, et al. Cigarette smoke extract reduces VEGF in primary human airway epithelial cells. Eur Respir J. 2009;33:835-43.

11. Berube K, Prytherch Z, Job C, et al. Human primary bronchial lung cell constructs: the new respiratory models. Toxicology. 2010;278:311-8.

12. Dvorak A, Tilley AE, Shaykhiev R, et al. Do airway epithelium air-liquid cultures represent the in vivo airway epithelium transcriptome? Am J Respir Cell Mol Biol. 2011;44:465-73.

13. Prytherch Z, Job C, Marshall H, et al. Tissue-Specific stem cell differentiation in an in vitro airway model. Macromol Biosci. 2011;11:1467-77.

14. Ostrowski LE, Stewart D, Hazucha M. Interferon gamma stimulates accumulation of gas phase nitric oxide in differentiated cultures of normal and cystic fibrosis airway epithelial cells. Lung. 2012;190:563-71.

15. Comer DM, Kidney JC, Ennis M, et al. Airway epithelial cell apoptosis and inflammation in COPD, smokers and nonsmokers. Eur Respir J. 2013;41:1058-67.

16. Damian S, Klarmann G, Smithhisler M. B-ALI(TM) Bronchial air liquid interface media kit, a guaranteed $3 \mathrm{D}$ in vitro model for respiratory research. Lonza Resource Notes 2010;Fall 2010: $13-16$.

17. Hao W, Bernard K, Patel N, et al. Infection and propagation of human rhinovirus $\mathrm{C}$ in human airway epithelial cells. J Virol. 2012;86:13524-32.

18. Hill DB, Button B. Establishment of respiratory air-liquid interface cultures and their use in studying mucin production, secretion, and function. Methods Mol Biol. 2012;842:245-58. 
19. Ong HX, Jackson CL, Cole JL, et al. Primary air-liquid interface culture of nasal epithelium for nasal drug delivery. Mol Pharm. 2016;13:2242-52.

20. Stevens PT, Kicic A, Sutanto EN, et al. Dysregulated repair in asthmatic paediatric airway epithelial cells: the role of plasminogen activator inhibitor-1. Clin Exp Allergy. 2008;38:1901-10.

21. Kicic A, Hallstrand TS, Sutanto EN, et al. Decreased fibronectin production significantly contributes to dysregulated repair of asthmatic epithelium. Am J Respir Crit Care Med. 2010;181: 889-98.

22. Barrow RE, Wang CZ, Evans MJ, et al. Growth factors accelerate epithelial repair in sheep trachea. Lung. 1993;171:335-44.

23. Takeyama K, Dabbagh K, Lee HM, et al. Epidermal growth factor system regulates mucin production in airways. Proc Natl Acad Sci USA. 1999;96:3081-6.

24. Schettino C, Bareschino MA, Ricci V, et al. Erlotinib: an EGF receptor tyrosine kinase inhibitor in non-small-cell lung cancer treatment. Expert Rev Respir Med. 2008;2:167-78.

25. Spann KM, Baturcam E, Schagen J, et al. Viral and host factors determine innate immune responses in airway epithelial cells from children with wheeze and atopy. Thorax. 2014;69:918-25.

26. Muller L, Brighton LE, Carson JL, et al. Culturing of human nasal epithelial cells at the air liquid interface. J Vis Exp. 2013;e50646: $1-7$.

27. Parker JC, Douglas I, Bell J, et al. Epidermal growth factor removal or tyrphostin AG1478 treatment reduces goblet cells \& mucus secretion of epithelial cells from asthmatic children using the air-liquid interface model. PLoS ONE. 2015;10:e0129546.

28. Zaidman NA, Panoskaltsis-Mortari A, O'Grady SM. Differentiation of human bronchial epithelial cells: role of hydrocortisone in development of ion transport pathways involved in mucociliary clearance. Am J Physiol Cell Physiol. 2016;311:C225-36.

29. Gras D, Chanez P, Vachier I, et al. Bronchial epithelium as a target for innovative treatments in asthma. Pharmacol Ther. 2013;140:290-305.

30. Comer DM, Elborn JS, Ennis M. Comparison of nasal and bronchial epithelial cells obtained from patients with COPD. PLoS ONE. 2012;7:e32924.

31. Rock JR, Randell SH, Hogan BL. Airway basal stem cells: a perspective on their roles in epithelial homeostasis and remodeling. Dis Model Mech. 2010;3:545-56.

32. Warner SM, Hackett TL, Shaheen F, et al. Transcription factor p63 regulates key genes and wound repair in human airway epithelial basal cells. Am J Respir Cell Mol Biol. 2013;49:978-88.

33. Wan H, Kaestner KH, Ang SL, et al. Foxa2 regulates alveolarization and goblet cell hyperplasia. Development. 2004;131: 953-64.

34. Wanner A, Salathe M, O'Riordan TG. Mucociliary clearance in the airways. Am J Respir Crit Care Med. 1996;154:1868-902.
35. Lu JJ, Xu GN, Yu P, et al. The activation of M3 mAChR in airway epithelial cells promotes IL-8 and TGF-beta1 secretion and airway smooth muscle cell migration. Respir Res. 2016;17:25.

36. Crosby LM, Waters CM. Epithelial repair mechanisms in the lung. Am J Physiol Lung Cell Mol Physiol. 2010;298:L715-731.

37. Gardner A, Borthwick LA, Fisher AJ. Lung epithelial wound healing in health and disease. Expert Rev Respir Med. 2010;4: 647-60.

38. Xiao H, Li DX, Liu M. Knowledge translation: airway epithelial cell migration and respiratory diseases. Cell Mol Life Sci. 2012;69:4149-62.

39. Grainge CL, Davies DE. Epithelial injury and repair in airways diseases. Chest. 2013;144:1906-12.

40. Zaja-Milatovic S, Richmond A. CXC chemokines and their receptors: a case for a significant biological role in cutaneous wound healing. Histol Histopathol. 2008;23:1399-407.

41. Arwert EN, Hoste E, Watt FM. Epithelial stem cells, wound healing and cancer. Nat Rev Cancer. 2012;12:170-80.

42. Vliagoftis H, Befus AD, Hollenberg MD, et al. Airway epithelial cells release eosinophil survival-promoting factors (GM-CSF) after stimulation of proteinase-activated receptor 2. J Allergy Clin Immunol. 2001;107:679-85.

43. Allahverdian S, Harada N, Singhera GK, et al. Secretion of IL-13 by airway epithelial cells enhances epithelial repair via HB-EGF. Am J Respir Cell Mol Biol. 2008;38:153-60.

44. Spurrell JCL, Wiehler S, Zaheer RS, et al. Human airway epithelial cells produce IP-10 (CXCL10) in vitro and in vivo upon rhinovirus infection. Am J Physiol-Lung Cell Mol Physiol. 2005;289:L85-95.

45. Tanaka T, Narazaki M, Kishimoto T. IL-6 in inflammation, immunity, and disease. Cold Spring Harb Perspect Biol. 2014;6: a016295.

46. Coraux C, Martinella-Catusse C, Nawrocki-Raby B, et al. Differential expression of matrix metalloproteinases and interleukin- 8 during regeneration of human airway epithelium in vivo. J Pathol. 2005;206:160-9.

47. Burgel PR, Nadel JA. Roles of epidermal growth factor receptor activation in epithelial cell repair and mucin production in airway epithelium. Thorax. 2004;59:992-6.

48. Prasad R, Giri S, Nath N, et al. Inhibition of phosphoinositide 3 kinase-Akt (protein kinase B)-nuclear factor-kappa B pathway by lovastatin limits endothelial-monocyte cell interaction. J Neurochem. 2005;94:204-14.

49. Jaspers I, Horvath KM, Zhang W, et al. Reduced expression of IRF7 in nasal epithelial cells from smokers after infection with influenza. Am J Respir Cell Mol Biol. 2010;43:368-75.

50. McDougall CM, Blaylock MG, Douglas JG, et al. Nasal epithelial cells as surrogates for bronchial epithelial cells in airway inflammation studies. Am J Respir Cell Mol Biol. 2008;39:560-8. 